\title{
Quantitative T2 Mapping Shows Increased Degeneration in Adjacent Intervertebral Discs Following Kyphoplasty
}

CARTILAGE

2020, Vol. II (2) I52-159

(c) The Author(s) 2018

Article reuse guidelines:

sagepub.com/journals-permissions

DOI: $10.1177 / 1947603518758434$

journals.sagepub.com/home/CAR

@SAGE

\author{
Milena L. Pachowsky ${ }^{1,2}$, Arnd Kleyer ${ }^{2,3}$, Lisa Wegener ${ }^{2}$, Andreas Langenbach ${ }^{1,2}$, \\ David Simon ${ }^{2,3}$, Rolf Janka ${ }^{2,4}$, Matthias May ${ }^{2,4}$, and Goetz H. Welsch ${ }^{5}$ iD
}

\begin{abstract}
Objective. A minimally invasive treatment of osteoporotic and nonosteoporotic thoracic and lumbar spine fractures is cement augmentation (kyphoplasty). Little is known about the impact on adjacent intervertebral discs. A quantitative magnetic resonance imaging (MRI) approach in addition to morphological MRI is desirable to evaluate changes in the intervertebral disc. Our study aims to evaluate the feasibility of T2 mapping for the detection of subtle changes in the intervertebral discs in spines after kyphoplasty. Design. Intervertebral discs were assessed by quantitative MRI (3.0 T) using T2 relaxation time mapping. Region of interest (ROI; 6 per disc) analyses were performed. The ROls at the anterior and posterior edges were interpreted as annulus fibrosus (AF). The 2 very inner zones were regarded as nucleus pulposus (NP) and the regions in between as intermediate transition zone. We compared T2 relaxation time values of intervertebral discs adjacent to the vertebrae after kyphoplasty with those nonadjacent to vertebrae after kyphoplasty, especially in the NP. Results. The analysis of the ROls showed that the intervertebral discs of the adjacent vertebral segments are associated with reduced $\mathrm{T} 2$ values compared to those that are nonadjacent to the affected vertebrae. Conclusion. This study is to our knowledge the first investigation of intervertebral discs after kyphoplasty by quantitative MRI. Quantitative T2 mapping shows increased degeneration in adjacent intervertebral discs following kyphoplasty. Besides its contribution to a broader knowledge of postoperative changes after kyphoplasty, our findings may help to improve differentiation between healthy and degenerated intervertebral discs using these techniques.
\end{abstract}

\section{Keywords}

kyphoplasty, T2 mapping, MRI, intervertebral disc, diagnosis

\section{Introduction}

In an aging society, fractures are a frequently observed cause of physical impairment and pain. ${ }^{1}$ Patients suffering from osteoporosis have a higher risk of fractures, particularly compression fractures in the thoracic and lumbar spine, and these vertebral compression fractures account for nearly as many cases as hip and distal radius osteoporotic fractures combined..$^{1-3}$ Besides reducing quality of life, they are associated with an increased risk of future vertebral and nonvertebral fractures. ${ }^{4}$ Vertebral augmentation is a minimally invasive procedure that aims to reduce pain and back-related disability by sustaining or restoring vertebral height and stabilizing osteoporotic and non-osteoporotic fractures. ${ }^{5}$ Percutaneous kyphoplasty is one of these vertebral augmentation procedures. Stabilizing the fracture with polymethylmethacrylate (PMMA) increases the density of the vertebral body. Little is known about the impact on the intervertebral disc. The annulus fibrosus (AF) of the intervertebral disc consists of fibrocartilage whereas in the nucleus pulposus (NP) loose fibers are embedded in a mucoprotein gel. Pathological discs can be differentiated from normal discs by changes in their water content and collagen arrangement. Healthy discs show lower water content in the AF than in the NP.

Standard magnetic resonance imaging (MRI) is a noninvasive and well-established method for the assessment of intervertebral discs and their morphologies. ${ }^{6}$ Increasing

\footnotetext{
'Department of Trauma and Orthopaedic Surgery, Universitätsklinikum Erlangen, Erlangen, Germany

${ }^{2}$ Friedrich-Alexander-University Erlangen-Nürnberg (FAU), Erlangen, Germany

${ }^{3}$ Department of Internal Medicine 3-Rheumatology and Immunology, Universitätsklinikum Erlangen, Erlangen, Germany

${ }^{4}$ Department of Radiology, Universitätsklinikum Erlangen, Erlangen, Germany

${ }^{5}$ UKE Athleticum, University Hospital Hamburg-Eppendorf, Hamburg, Germany
}

Corresponding Author:

Goetz H. Welsch, UKE Athleticum, University Hospital HamburgEppendorf, Building East 48, Martinistraße 52, 20246 Hamburg, Germany.

Email: g.welsch@uke.de 
field strength and higher spatial resolution MRI helps detect subtle changes in the vertebral body and in the intervertebral disc. To differentiate between NP and AF, T2-weighted MR sequences are applied. ${ }^{7}$ However, these images provide only qualitative data. Quantitative imaging methods aim to visualize the ultrastructure of tissues. T2 mapping visualizes variations in collagen orientation and water mobility and hence is an attempt to obtain information about tissue composition by calculating relaxation times; it has the potential to identify various grades of cartilage damage. ${ }^{8,9}$ Our study aims to evaluate the feasibility of T2 mapping for detecting subtle changes in the intervertebral discs in spines operatively treated by kyphoplasty.

\section{Material and Methods}

Ethical approval for this study was provided by the Clinical Ethics Committee (CEC) of the local university.

An average of $25.8 \pm 5.2$ months passed between the surgical procedure and the postoperative biochemical-based MRI. We selected our study population from a group of 73 subjects who had suffered from fractures of the lumbar or lower thoracal spine and were treated by kyphoplasty. The indication for this vertebral augmentation procedure was defined on the basis of the results of clinical examination and diagnostic imaging.

In all patients, kyphoplasty was performed under general anesthesia in a prone position. Needles and balloons were inserted into the vertebral body from posterior passing through the vertebral arch by biportal access. The vertebrae were then inflated by the balloons and after removal of the balloons filled with PMMA. All cement augmentations used fluoroscopically controlled needle placement to achieve an optimal result of balloon fracture reduction and cement application. ${ }^{10}$ Patients who suffered from subsequent vertebral fractures between the date of surgery and the second examination point were excluded. Further exclusion criteria were the presence of higher grades of disc herniation, immobility, and severe comorbidity. Sixteen patients with claustrophobia had to be excluded. Of the remaining patients, 14 gave their informed consent to participate in this study.

We included these remaining 14 patients ( 9 females, 5 males; mean age $64.5 \pm 7.53$ years) in the study.

\section{Magnetic Resonance Imaging}

A 3 T MR scanner (Magnetom Skyra, Siemens Medical Solutions, Erlangen, Germany) with a gradient strength of $40 \mathrm{mT} / \mathrm{m}$ and a dedicated 8-channel spine coil (3 T Spine Matrix Coil, Siemens) were used for image acquisition. In order to standardize loading forces, patients had to rest in a sitting position for 30 minutes prior to $\mathrm{T} 2$ mapping examination. Thereafter, images were acquired while patients were in a supine position. The protocol stipulated sagittal, multiecho, spin-echo (SE) T2 image acquisition. For T2 relaxation time measurement, a multi-echo-spin-echo sequence with a repetition time (TR) of $1400 \mathrm{~ms}$, echo times (TE) of 11.9, 23.8, 35.7, 47.6, 59.5, 71.4, 83.3, 95.2, 107.1, 119, 130.9 , and $142.8 \mathrm{~ms}$ was used. The field of view (FOV) was $260 \times 260$ with a flip angle of $180^{\circ}$. The pixel size was $0.4 \times$ $0.4 \times 5.0 \mathrm{~mm}$, slice thickness $5 \mathrm{~mm}$, bandwidth $200 \mathrm{~Hz} /$ pixel and the scan time was $6: 18$ minutes. T2 relaxation times were obtained from on-line reconstructed $\mathrm{T} 2$ maps using a pixel-wise, mono-exponential, nonnegative least squares (NNLS) fit analysis (MapIt, Siemens Medical Solutions, Erlangen, Germany). High-resolution morphological MRI was also performed using a 3-dimensional, isotropic, fatsaturated proton-density space sequence (PD-SPACE). All sequences were acquired in the sagittal plane. For image acquisition, the vertebral bodies and the processus spinosus were used as anatomical landmarks. In the PD-SPACE sequence, the imaging specialists defined the regions of interest (ROIs) and copied them onto the T2 map. This step was considered critical for precise localization of the defined ROIs in the $\mathrm{T} 2$ sequence images. This clear form of orientation provided the basis for the subsequent image analysis.

\section{Image Analysis}

For ROI analysis, the MRI data sequences were transferred to a Syngo (Leonardo) workstation (Siemens Medical Solution, Erlangen, Germany). T2 relaxation time maps were obtained using a NNLS fit analysis. First, the imaging specialists (M.P., G.W.) examined the morphological data sets for any new fractures or motion artifacts. Assessment of intervertebral discs between Th 12 and S1 was performed. ROIs were defined in 4 sagittal planes: 2 through the right and 2 through the left portion of the vertebral disc. The anterior and posterior rims of the vertebral bone were used as anatomical landmarks. Vertebral disc status was assessed in these 4 planes (see Figures 1 and 2). For subregional analysis, we marked 6 regions within the vertebral disc. The gradual transition from AF to NP tissue often causes difficulties in defining a clear boundary. In order to evaluate the ROIs in a reproducible manner, these 6 regions were measured by equally sized "squared" ROIs in 4 adjacent slices on sagittal T2 maps. The ROIs at the anterior and posterior edges (ROI 1 and ROI 6) were interpreted as AF tissues (Fig. 1, green squares). Regions 2 and 5 were regarded as an intermediate transition zone between $\mathrm{AF}$ and NP (Fig. 1, orange squares). In between these anterior and posterior regions, the ROIs 3 and 4 were defined as definite nucleus pulposus tissue (Fig. 1, red squares).

The discs were grouped according to whether they presented with contact to a fractured and post-kyphoplasty vertebra or with contact to "normal" vertebrae. Group 1 comprises intervertebral discs adjacent to vertebrae after kyphoplasty. 


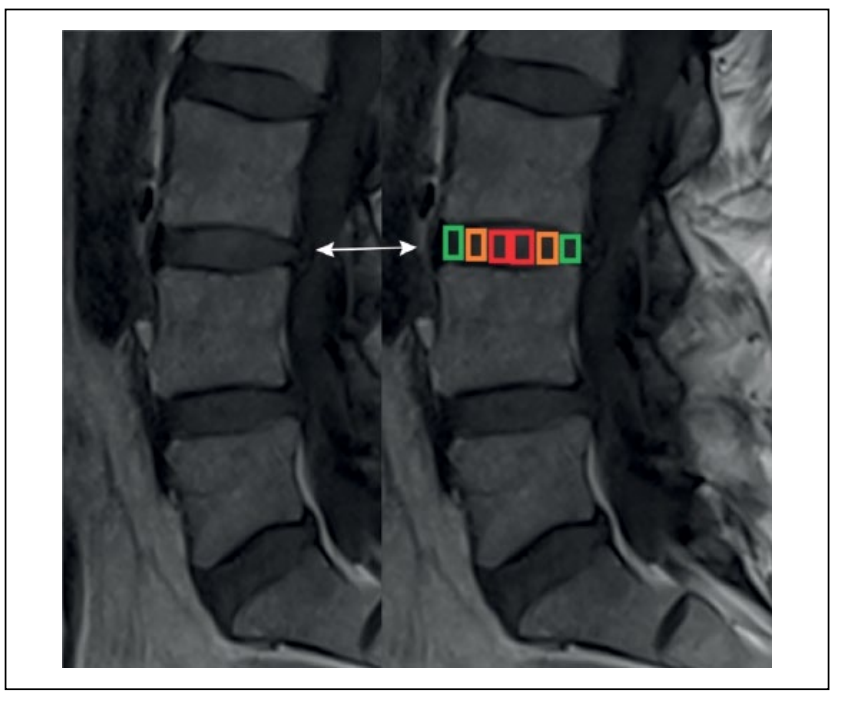

Figure I. Schematic overview of region of interest (ROI) subdivision of the intervertebral disc. The ROls at the anterior and posterior edges (ROI I and ROI 6) were interpreted as annulus fibrosus (AF) tissue (green squares). Regions 2 and 5 were seen as intermediate transition zone between $A F$ and nucleus pulposus (orange squares). In between these most anterior and posterior regions, ROls 3 and 4 were defined as definite nucleus pulposus tissue (red squares) (For interpretation of the references to colours in this figure legend, refer to the online version of this article).

Group 2 comprises intervertebral discs adjacent to vertebrae without kyphoplasty.

\section{Statistical Analysis}

Data were collected, organized, and analyzed through SPSS software for statistics (IBM SPSS 21.0, IBM Corp., Armonk, NY, USA). If not stated otherwise, continuous variables are provided as mean and standard deviations (SD).

For the comparison of T2 relaxation time values between adjacent and nonadjacent intervertebral discs (for each ROI) the following procedure was performed: assumptions of normally distributed continuous variables were tested using quantile-quantile plots, Kolmogorov-Smirnov, and Shapiro-Wilk tests. After testing for Gaussian distribution, the Mann-Whitney $U$ test or $t$ test for independent samples was applied as appropriate.

$P$ values $\leq 0.05$ were considered as statistically significant.

\section{Results}

\section{Patients' Characteristics and ROls}

Patients had a mean age of $64.5 \pm 7.53$ years when they were examined by MRI. The mean body weight in our cohort was $76.50 \pm 14.46 \mathrm{~kg}$ with an average body mass index of $26.66 \mathrm{~kg} / \mathrm{m}^{2}$. The time between surgery and MRI was on average $25.79 \pm 5.16$ months.
Quantitative T2 mapping for intervertebral disc fibrocartilage assessment was performed in 14 patients. A total of 504 ROIs (36 per spine) were analyzed. The mean numbers of pixels for the ROIs are provided in Table 1.

We compared mean T2 values between adjacent and nonadjacent intervertebral discs for each ROI (1-6) in each of the segments evaluated (Th12/L1- L5/S1).

\section{Results According to the Anatomical Distribution of the ROls within the Intervertebral Disc}

Outer Part (AF). ROIs 1 and 6 represent the outer parts of the intervertebral disc (Fig. 1, green squares). Only T2 relaxation time values for segment Th12/L1 in ROI 1 showed a statistically significant difference between nonadjacent and adjacent vertebral discs (adjacent $62.62 \pm 51.77$ vs. nonadjacent $45.82 \pm 16.36 ; P=0.005)$ whereas all other ROIs 1 and 6 did not reveal any significant differences with divergent results, (see Table 2 ).

Intermediate Zone (IZ). ROIs 2 and 5 represent the intermediate zone (Fig. 1, orange squares).

Only values for segments L1/L2 and L2/3 showed lower T2 values in the adjacent intervertebral discs of ROIs 2 with a statistical significant difference. In segment Th12/L1 the values were divergent, with higher values in adjacent discs, but again a statistically significant difference was observed.

Looking at ROI 5 we could see statistically significantly lower T2 values in the adjacent intervertebral discs in segments L2/L3 and L4/L5. In segment L1/L2, the values were divergent, with higher values in adjacent discs, and a statistically significant difference could be observed again. In all other ROIs 2 and 5 of all segments analyzed, adjacent intervertebral discs showed lower mean values, but did not reveal any statistically significant differences.

Inner Part (NP). ROIs 3 and 4 comprise the inner part of the intervertebral disc representing the NP (Fig. 1, red squares). We could detect decreasing T2 values in the adjacent discs compared to the nonadjacent intervertebral discs in segments $\mathrm{L} 1 / \mathrm{L} 2$ to $\mathrm{L} 3 / \mathrm{L} 4$. In these 6 ROIs, representing the very inner part of the intervertebral disc of the upper lumbar spine, the adjacent discs showed statistically significantly lower T2 values than the nonadjacent intervertebral discs, for ROI $3 \mathrm{~L} 1 / \mathrm{L} 2$ adjacent $60.49 \pm 13.88$ versus nonadjacent $66.07 \pm 33.12, P<0.01$; L2/L3 adjacent $49.13 \pm 13.04$ versus nonadjacent $73.18 \pm 33.73, P<0.01$; L3/L4 adjacent $53.76 \pm 9.04$ versus nonadjacent $67.90 \pm 16.57, P<0.01$ and for ROI $4 \mathrm{~L} 1 / \mathrm{L} 2$ adjacent $57.92 \pm 12.70$ versus nonadjacent $62.60 \pm 21.72, P<0.01 ; \mathrm{L} 2 / \mathrm{L} 3$ adjacent $52.1 \pm 11.67$ versus nonadjacent $75.63 \pm 36.24, P<0.01$; L3/L4 adjacent $51.92 \pm 10.85$ versus nonadjacent $71.01 \pm 24.46, P<0.01$.

For segments L4/L5 and L5/S1, nonadjacent intervertebral discs showed higher values in all ROIs, but the 

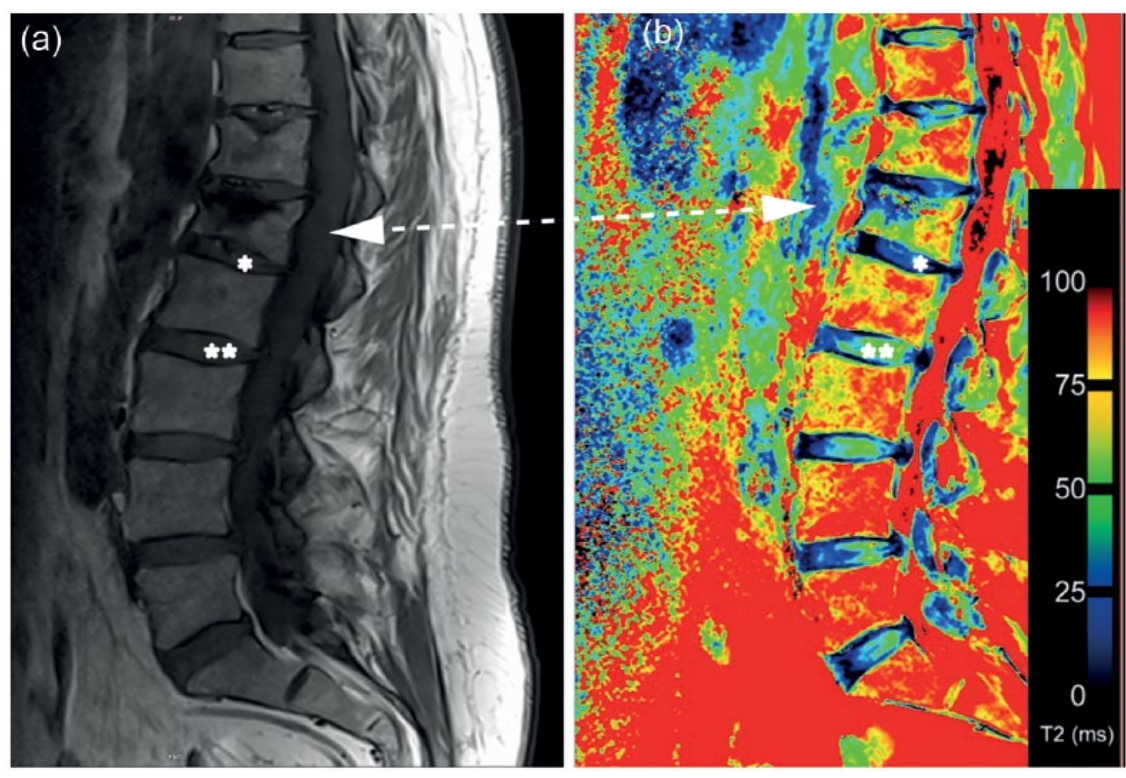

Figure 2. (a) Morphological magnetic resonance image of the lumbar spine. Vertebral body after kyphoplasty (LI) marked by arrow. Intervertebral discs adjacent to vertebral body after kyphoplasty marked with an asterisk (*), intervertebral discs nonadjacent to vertebral body after kyphoplasty marked with double asterisks (**). (b) T2 map with clearly visible differences between intervertebral discs adjacent $(*)$ and nonadjacent $(* *)$ to vertebral bodies after kyphoplasty.

Table I. Mean Number of Pixels for Each Region of Interest (ROI) in Every Segment.

\begin{tabular}{lllllll}
\hline & \multicolumn{6}{c}{ Pixels (mean number) } \\
\cline { 2 - 7 } Localization & ROI I & ROI 2 & ROI 3 & ROI 4 & ROI 5 & ROI 6 \\
\hline ThI2/LI & 263.70 & 287.12 & 317.54 & 326.34 & 286.79 & I69.II \\
LI/L2 & 337.77 & 365.55 & 394.71 & 384.28 & $340.4 I$ & 211.41 \\
L2/L3 & 360.13 & 405.59 & 456.63 & $46 I .18$ & 395.13 & 237.43 \\
L3/L4 & 388.86 & 448.20 & 529.04 & $46 I .18$ & 504.55 & 422.86 \\
L4/L5 & 462.21 & 452.50 & 476.70 & 454.63 & 383.11 & 247.75 \\
L5/SI & 413.20 & 353.45 & 363.32 & 330.43 & 290.75 & 192.02 \\
\hline
\end{tabular}

differences were not significant. For all mean T2 and $P$ values, see Table 2.

Interestingly, in most of the evaluated ROIs between L1/ $\mathrm{L} 2$ and L5/S1, mean T2 values were noticeably higher in the nonadjacent intervertebral discs, although not all of them were statistically significantly different. In almost all ROIs (5/6) of segment Th12/L1 the reverse distribution was observable, and $\mathrm{T} 2$ values of the intervertebral discs in the adjacent group were higher than in the nonadjacent discs. Mean T2 values are visualized in Figure 3.

To summarize the values detected, the analysis of the ROIs showed that in our cohort, intervertebral discs of the NP in segments L1/2 to L3/L4 develop lower T2 values in the discs adjacent to vertebrae after kyphoplasty compared with those nonadjacent to vertebrae after kyphoplasty. Figure 2b shows a representative T2 mapping image of adjacent and nonadjacent discs, with clearly visible differ- ences between intervertebral discs adjacent and nonadjacent to vertebral bodies after kyphoplasty.

\section{Discussion}

This study investigated intervertebral discs approximately 2 years after kyphoplasty, focusing on differences between adjacent and nonadjacent discs. Quantitative T2 mapping was used to assess changes in the fibrocartilage substructure.

Our findings showed a significant decrease in $\mathrm{T} 2$ relaxation times in the mid-portion of the intervertebral discs adjacent to vertebral bodies after kyphoplasty. These findings could be an interesting basis for further studies and support the hypothesis that varying statics after vertebral augmentation procedures might have an influence on the adjacent intervertebral discs.

Increasing life expectancy in modern society will lead to a higher prevalence of osteoporotic fractures, meaning that numbers of intervertebral disc augmentation procedures will rise. It is important to evaluate concomitants of these procedures in order to be prepared for possible complications or impact on the patient's life afterward.

Standard morphological MRI has become an accepted diagnostic tool for non-invasive evaluation of various musculoskeletal alterations, for example, bone bruise, intervertebral disc or cartilage lesions.

Moreover, emerging techniques, including T2 mapping, can be used to visualize microstructural and biochemical changes to the soft tissue matrix. 
Table 2. T2 Relaxation Time Values (Mean \pm SD) for Regions of Interest (ROls) I and 6 (Upper Third of the Table) Representing Annulus Fibrosus Tissue (AF); for the ROls 2 and 5 (Middle Third of the Table), Representing the Intermediate Zone (IZ); and for the ROIs 3 and 4, Representing Nucleus Pulposus Tissue (NP) Subcategorized by the Segments ThI2/LI to L5/SI and P Values for the Difference between Adjacent and Nonadjacent Intervertebral Discs. ${ }^{a}$

\begin{tabular}{|c|c|c|c|c|c|c|}
\hline \multirow[b]{2}{*}{ Localization } & \multicolumn{3}{|c|}{ ROI I } & \multicolumn{3}{|c|}{ ROI 6} \\
\hline & Adjacent & Nonadjacent & $P$ Value Difference & Adjacent & Nonadjacent & $P$ Value Difference \\
\hline \multicolumn{7}{|l|}{$\mathrm{AF}$} \\
\hline ThI2/LI & $62.62 \pm 51.77$ & $45.82 \pm 16.36$ & 0.005 & $64.76 \pm 35.53$ & $74.95 \pm 80.07$ & 0.368 \\
\hline $\mathrm{LI} / \mathrm{L} 2$ & $52.76 \pm 14.77$ & $56.91 \pm 24.34$ & 0.913 & $54.37 \pm 22.91$ & $56.55 \pm 26.14$ & 0.097 \\
\hline L2/L3 & $55.35 \pm 17.59$ & $64.24 \pm 38.57$ & 0.689 & $45.66 \pm 11.13$ & $58.35 \pm 31.26$ & 0.097 \\
\hline L3/L4 & $57.21 \pm 32.87$ & $69.34 \pm 49.43$ & 0.065 & $50.38 \pm 13.37$ & $61.20 \pm 43.55$ & 0.577 \\
\hline L4/L5 & $53.65 \pm 14.78$ & $49.80 \pm 15.77$ & 0.246 & $60.05 \pm 17.75$ & $55.11 \pm 17.62$ & 0.282 \\
\hline \multirow[t]{3}{*}{ L5/SI } & $49.36 \pm 8.40$ & $49.94 \pm 20.95$ & 0.383 & $64.31 \pm 21.69$ & $74.12 \pm 63.63$ & 0.700 \\
\hline & \multicolumn{3}{|c|}{ ROI 2} & \multicolumn{3}{|c|}{ ROI 5} \\
\hline & Adjacent & Nonadjacent & $P$ Value Difference & Adjacent & Nonadjacent & $P$ Value Difference \\
\hline \multicolumn{7}{|l|}{ IZ } \\
\hline ThI2/LI & $52.82 \pm 16.86$ & $39.03 \pm 7.99$ & 0.002 & $58.35 \pm 18.17$ & $54.96 \pm 11.47$ & 0.766 \\
\hline LI/L2 & $50.46 \pm 10.95$ & $61.13 \pm 42.24$ & 0.000 & $49.67 \pm 13.24$ & $40.1 \pm 13.3$ & 0.000 \\
\hline L2/L3 & $43.94 \pm 11.56$ & $64.44 \pm 44.22$ & 0.000 & $45.54 \pm 9.89$ & $60.38 \pm 12.93$ & 0.000 \\
\hline L3/L4 & $54.86 \pm 18.70$ & $57.85 \pm 15.70$ & 0.285 & $47.41 \pm 8.01$ & $58.04 \pm 12.64$ & 0.002 \\
\hline L4/L5 & $53.01 \pm 12.75$ & $53.96 \pm 11.62$ & 0.547 & $54.58 \pm 4.65$ & $57.36 \pm 9.39$ & 0.318 \\
\hline \multirow[t]{3}{*}{ L5/SI } & $56.38 \pm 8.40$ & $55.72 \pm 15.98$ & 0.839 & $55.28 \pm 16.15$ & $64.66 \pm 18.59$ & 0.128 \\
\hline & \multicolumn{3}{|c|}{ ROI 3} & \multicolumn{3}{|c|}{ ROI 4} \\
\hline & Adjacent & Nonadjacent & $P$ Value Difference & Adjacent & Nonadjacent & $P$ Value Difference \\
\hline \multicolumn{7}{|l|}{ NP } \\
\hline ThI2/LI & $64.56 \pm 30.12$ & $55.69 \pm 10.82$ & 0.457 & $65.09 \pm 23.56$ & $53.35 \pm 14.78$ & 0.535 \\
\hline $\mathrm{LI} / \mathrm{L} 2$ & $60.49 \pm 13.88$ & $66.07 \pm 33.12$ & 0.000 & $57.92 \pm 12.70$ & $62.60 \pm 21.72$ & 0.000 \\
\hline L2/L3 & $49.13 \pm 13.04$ & $73.18 \pm 33.73$ & 0.000 & $52.1 \pm 11.67$ & $75.63 \pm 36.24$ & 0.000 \\
\hline L3/L4 & $53.76 \pm 9.04$ & $67.90 \pm 16.57$ & 0.002 & $51.92 \pm 10.85$ & $71.01 \pm 24.46$ & 0.001 \\
\hline L4/L5 & $55.76 \pm 9.13$ & $61.71 \pm 14.78$ & 0.177 & $55.20 \pm 7.29$ & $62.81 \pm 13.71$ & 0.063 \\
\hline L5/SI & $65.83 \pm 31.12$ & $70.25 \pm 29.02$ & 0.440 & $56.46 \pm 20.06$ & $66.38 \pm 23.79$ & 0.092 \\
\hline
\end{tabular}

${ }^{\text {a }} P$ values $\leq 0.05$ are marked in boldface.

Conventional T2-weighted sagittal MRI sequences have been used to create a subjective grading scale for disc health based on morphological criteria. ${ }^{11,12}$ The most discussed potential problem with the grading system is its lack of specificity. ${ }^{13,14}$ The Pfirrmann grading system is based on MRI signal intensity, the clarity of the transition zone between the AF and NP, and disc height. ${ }^{11}$ Several studies have broached the issue of this subjective scoring system's failure to correlate well with pain or provide clinically useful patient stratification, especially in detecting early signs of degeneration. ${ }^{15,16}$

Ellingson et al. ${ }^{17}$ showed that $\mathrm{T} 2 *$ mapping is a sensitive quantitative method capable of detecting changes associated with disc degeneration and predicting altered functional mechanics of the lumbar spine (in cadaveric measurements) better than traditional Pfirrmann grading. Numerous previous studies have compared quantitative imaging methods and assessment in morphological sequences by Pfirrmann grading. ${ }^{18}$ As these studies have demonstrated correlation between the methodologies, we decided to omit grading according to the Pfirrmann score.

In our study, we intended to investigate intervertebral discs after kyphoplasty by quantitative MR and to compare the findings in adjacent and nonadjacent discs. Analysis of the ROIs showed that in our cohort, intervertebral discs of the NP develop lower T2 values in the discs adjacent to vertebrae after kyphoplasty compared with those nonadjacent to vertebrae after kyphoplasty. These findings underscore the ability of T2 mapping to detect subtle changes in vertebral discs after kyphoplasty, and our initial results suggest that there are differences in the ultrastructure of intervertebral discs after kyphoplasty. Our hypothesis was that the adjacent intervertebral discs tend to develop accelerated degenerative changes. The decrease in mean $\mathrm{T} 2$ values in 

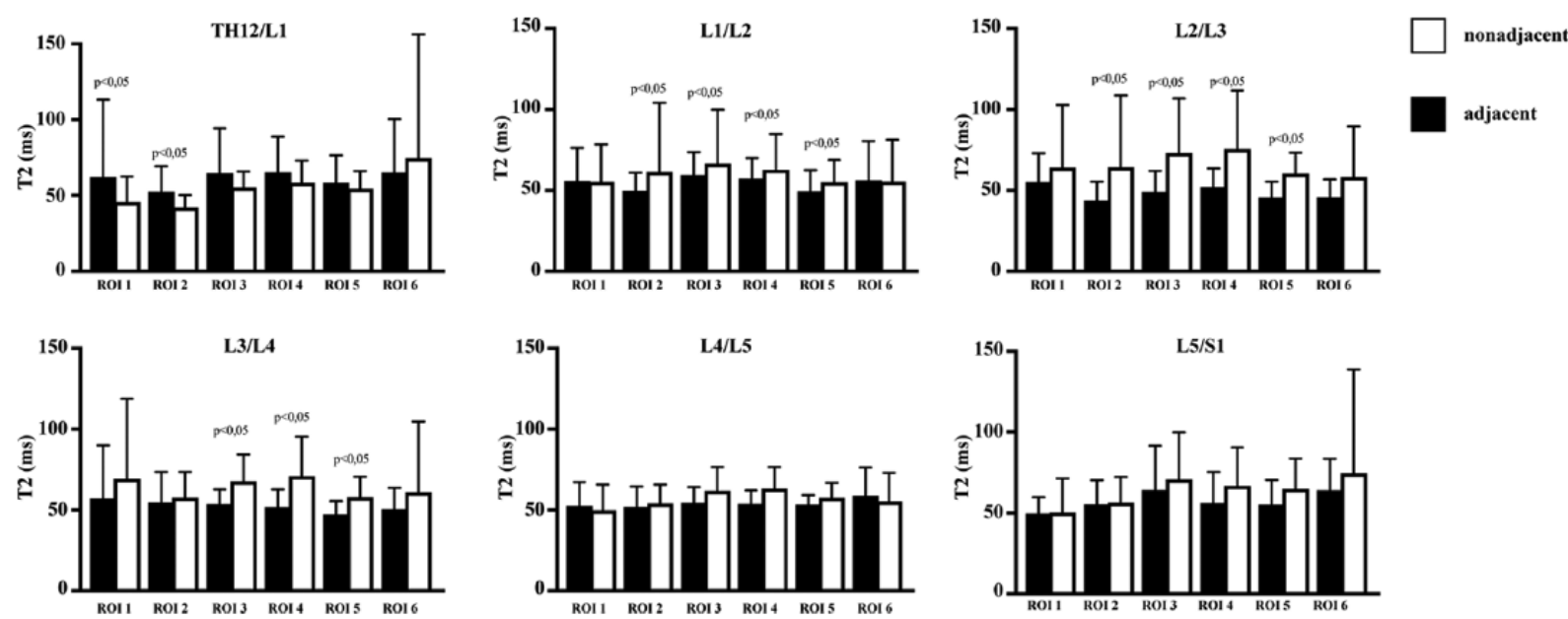

Figure 3. Average $T 2$ relaxation time values for the segments $T h I 2 / L I$ to $L 5 / S I$. For each region of interest (ROI), black bars display data of adjacent intervertebral discs, white bars display data of nonadjacent intervertebral discs. Statistically significant differences between adjacent and nonadjacent intervertebral discs are marked with $P \leq 0.05$.

the mapping analysis we have presented here is congruent with the findings of Niinimäki et al., ${ }^{19}$ who correlated degeneration with dehydration in an animal experiment, and explainable by the findings of Marinelli et al., ${ }^{20}$ who reported that quantitative $\mathrm{T} 2$ values correlated significantly with the water content in the human NP. Compared with these results of previous studies, our investigation confirmed the presence of partly morphologically visible degenerative changes in the intervertebral disc. The subregional mapping analysis will help us understand changes in the tissue after kyphoplasty.

The anterior portion of the intervertebral disc (ROI 1) and the posterior edge (ROI 6) do not seem to be influenced particularly by the presence of kyphoplasty. The tendency for significantly lower $\mathrm{T} 2$ average values in the adjacent intervertebral discs within ROIs 2 to 5 clearly suggests that the inner portion of the intervertebral disc is influenced by the presence of kyphoplasty. Although we did not analyze the distribution of the cement within the vertebral body, we could observe a clear pattern after kyphoplasty in segments L1/L2 to L3/4 in the inner part of the disc. This pattern might give a hint that the impact of kyphoplasty on the intervertebral discs is most marked in the apex of the lumbar lordosis.

König et $_{\text {al }}{ }^{21}$ found no significant influence on disc degeneration at an average of 15.2 months after kyphoplasty. Their results are in contrast to our findings. Our cohort was investigated a little over two years after kyphoplasty, hence at a later postoperative stage than their patients on average. Their imaging approach used morphological imaging. Two factors could explain our differing results. The first is our longer postoperative interval (15 vs. 25 months), which may lead to further progression of the degenerative changes. The second is the imaging method we used, which enabled us to detect more subtle changes in the intervertebral discs. An additional factor could be differing preoperative conditions. This new methodology and analysis technique may enhance the assessment of degeneration and could be helpful for the assessment of patients with persisting pain after kyphoplasty.

Adjacent segment degeneration has been reported to be likely after lumbar fusion surgeries. Moreau et al $^{22}$ reported that $29 \%$ of patients developed adjacent segment degeneration, which was mainly associated with risk factors such as a high number of instrumented levels and preoperative sagittal imbalance. In their survey on the effects of fusion, D'Oro $\mathrm{et}_{\mathrm{al}}{ }^{23}$ found that lumbar fusion surgery increases the incidence of disc degeneration. Rohlmann et al. ${ }^{24}$ compared the effects of posterior dynamic and rigid fixation devices on the loads in the lumbar spine in a finite element study. They showed that the mechanical effects of dynamic implants are comparable to those of a rigid fixation device in the adjacent level (here solely the segment L2/L3 was analyzed), except after distraction, when intradiscal pressure is considerably lower for rigid than for dynamic implants. ${ }^{24}$ However, the mechanism at work after kyphoplasty might be different from that found in these settings, due to the fact that mobility between the segments is not directly influenced by cement augmentation. Therefore, the increased density of the vertebra after kyphoplasty might even lead to higher compression forces on the intervertebral disc. Ottardi et al. ${ }^{25}$ observed pronounced variations of intradiscal pressures and stresses after kyphoplasty. Most biomechanical studies focus on the biomechanical effect on the adjacent vertebrae and not on the intervertebral disc. ${ }^{26,27}$ Our study aimed to supply a first step toward a biochemical and biomechanical imaging approach to intervertebral discs after kyphoplasty. 
There are some limitations in the present study. One of these limitations is the lack of a gold standard. Understandably, no histological samples could be obtained.

Because of the fact that the patients in the investigated cohort were advanced in years, it was extremely challenging to find a control group with completely healthy spines. Therefore, we decided to use the intervertebral discs nonadjacent to vertebrae after kyphoplasty as an "internal" control group. However, future studies will need to be based on larger cohorts and include healthy controls in the study population. Longitudinal data will be needed to detect possible differences between female and male patients. In future studies, one of the aims will be to examine larger cohorts over a longer period of time. The lack of several follow-up scans is a limitation.

Another limitation of our study is the relatively small cohort. Future studies with larger patient groups will be needed to confirm the results. Additionally, clinical factors should be taken into account and will be correlated with the imaging findings. Another interesting task of future studies might be to investigate the endplate diffusion of the affected disc and to determine which role this condition plays and whether this might be a target for interventions. Future studies will additionally need to explore the role of the magic angle in $\mathrm{T} 2$ relaxation time mapping, as this might be a significant factor, especially in patients with pronounced lordosis or kyphosis.

Our initial study investigating T2 mapping values of intervertebral discs in spines after kyphoplasty could be a step toward a better understanding of clinical results and toward establishing well-evaluated follow-up measurements by biochemical and morphological MRI.

\section{Conclusion}

This study is, to our knowledge, the first investigation of intervertebral discs after kyphoplasty by quantitative MRI. Besides its contribution to a broader knowledge of postoperative changes after kyphoplasty, our findings may help to improve differentiation between healthy and degenerated intervertebral discs using these techniques.

\section{Authors' Note}

The work reported was done at Friedrich-Alexander-University Erlangen-Nürnberg, Erlangen, Germany.

\section{Acknowledgments and Funding}

The author(s) received no financial support for the research, authorship, and/or publication of this article.

\section{Declaration of Conflicting Interests}

The author(s) declared no potential conflicts of interest with respect to the research, authorship, and/or publication of this article.

\section{Ethical Approval}

Ethical approval for this study was provided by the Clinical Ethics Committee (CEC) of the local university (approval number 298_16 B).

\section{Informed Consent}

Written informed consent was obtained from all subjects before the study.

\section{Trial Registration}

Not applicable.

\section{ORCID iD}

Goetz H. Welsch (iD https://orcid.org/0000-0003-3140-3980

\section{References}

1. Amin S, Achenbach SJ, Atkinson EJ, Khosla S, Melton LJ 3rd. Trends in fracture incidence: a population-based study over 20 years. J Bone Miner Res. 2014;29:581-9.

2. Burge R, Dawson-Hughes B, Solomon DH, Wong JB, King A, Tosteson A. Incidence and economic burden of osteoporosis-related fractures in the United States, 2005-2025. J Bone Miner Res. 2007;22:465-75.

3. Orsini LS, Rousculp MD, Long SR, Wang S. Health care utilization and expenditures in the United States: a study of osteoporosis-related fractures. Osteoporos Int. 2005;16:359-71.

4. Ross PD. Clinical consequences of vertebral fractures. Am J Med. 1997;103:30S-43S.

5. Ensrud KE, Schousboe JT. Clinical practice. Vertebral fractures. N Engl J Med. 2011;364:1634-42.

6. Modic MT, Ross JS. Lumbar degenerative disk disease. Radiology. 2007;245:43-61.

7. Stelzeneder D, Welsch GH, Kovacs BK, Goed S, PaternostroSluga T, Vlychou M, et al. Quantitative T2 evaluation at 3.0 T compared to morphological grading of the lumbar intervertebral disc: a standardized evaluation approach in patients with low back pain. E J Radiol. 2012;81:324-30.

8. Bittersohl B, Hosalkar HS, Miese FR, Schibensky J, König $\mathrm{DP}$, Herten $\mathrm{M}$, et al. Zonal T2* and T1Gd assessment of knee joint cartilage in various histological grades of cartilage degeneration: an observational in vitro study. BMJ Open. 2015;5:e006895.

9. Eagle S, Potter HG, Koff MF. Morphologic and quantitative magnetic resonance imaging of knee articular cartilage for the assessment of post-traumatic osteoarthritis. J Orthop Res. 2017;35:412-23.

10. Wenger M, Markwalder TM. Surgically controlled, transpedicular methyl methacrylate vertebroplasty with fluoroscopic guidance. Acta Neurochir (Wien). 1999;141:625-31.

11. Pfirrmann CW, Metzdorf A, Zanetti M, Hodler J, Boos N. Magnetic resonance classification of lumbar intervertebral disc degeneration. Spine (Phila Pa 1976). 2001;26:1873-8.

12. Thompson JP, Pearce RH, Schechter MT, Adams ME, Tsang IK, Bishop PB. Preliminary evaluation of a scheme for grading the gross morphology of the human intervertebral disc. Spine (Phila Pa 1976). 1990;15:411-5. 
13. Arana E, Royuela A, Kovacs FM, Estremera A, Sarasibar H, Amengual G, et al. Lumbar spine: agreement in the interpretation of 1.5-T MR images by using the Nordic Modic Consensus Group classification form. Radiology. 2010;254:809-17.

14. Raininko R, Manninen H, Battie MC, Gibbons LE, Gill K, Fisher LD. Observer variability in the assessment of disc degeneration on magnetic resonance images of the lumbar and thoracic spine. Spine (Phila Pa 1976). 1995;20: 1029-35.

15. Jensen MC, Brant-Zawadzki MN, Obuchowski N, Modic MT, Malkasian D, Ross JS. Magnetic resonance imaging of the lumbar spine in people without back pain. N Engl J Med. 1994;331:69-73.

16. Powell MC, Wilson M, Szypryt P, Symonds EM, Worthington BS. Prevalence of lumbar disc degeneration observed by magnetic resonance in symptomless women. Lancet. 1986;2: 1366-7.

17. Ellingson AM, Mehta H, Polly DW, Ellermann J, Nuckley DJ. Disc degeneration assessed by quantitative T2* (T2 star) correlated with functional lumbar mechanics. Spine (Phila Pa 1976). 2013;38:E1533-E1540.

18. Welsch GH, Trattnig S, Paternostro-Sluga T, Bohndorf K, Goed S, Stelzeneder D, et al. Parametric T2 and T2* mapping techniques to visualize intervertebral disc degeneration in patients with low back pain: initial results on the clinical use of 3.0 tesla MRI. Skeletal Radiol. 2011;40:543-51.

19. Niinimäki J, Ruohonen J, Silfverhuth M, Lappalainen A, Kääpä E, Tervonen O. Quantitative magnetic resonance imaging of experimentally injured porcine intervertebral disc. Acta Radiol. 2007;48:643-9.
20. Marinelli NL, Haughton VM, Muñoz A, Anderson PA. T2 relaxation times of intervertebral disc tissue correlated with water content and proteoglycan content. Spine (Phila Pa 1976). 2009;34:520-4.

21. König MA, Panzer S, Schulz J, Bierschneider M, Boszczyk BM. Magnetic resonance imaging changes of intervertebral discs after kyphoplasty. Eur Spine J. 2015;24:724-33.

22. Moreau PE, Ferrero E, Riouallon G, Lenoir T, Guigui P. Radiologic adjacent segment degeneration 2 years after lumbar fusion for degenerative spondylolisthesis. Orthop Traumatol Surg Res. 2016;102:759-63.

23. D'Oro A, Spoonamore MJ, Cohen JR, Acosta FL, Hsieh PC, Liu JC, et al. Effects of fusion and conservative treatment on disc degeneration and rates of subsequent surgery after thoracolumbar fracture. J Neurosurg Spine. 2016;24:476-82.

24. Rohlmann A, Burra NK, Zander T, Bergmann G. Comparison of the effects of bilateral posterior dynamic and rigid fixation devices on the loads in the lumbar spine: a finite element analysis. Eur Spine J. 2007;16:1223-31.

25. Ottardi C, La Barbera L, Pietrogrande L, Villa T. Vertebroplasty and kyphoplasty for the treatment of thoracic fractures in osteoporotic patients: a finite element comparative analysis. J Appl Biomater Funct Mater. 2016;14:e197-e204.

26. Dabirrahmani D, Becker S, Hogg M, Appleyard R, Baroud G, Gillies M. Mechanical variables affecting balloon kyphoplasty outcome - a finite element study. Comput Methods Biomech Biomed Engin. 2012;15:211-20.

27. Rohlmann A, Zander T, Bergmann G. Spinal loads after osteoporotic vertebral fractures treated by vertebroplasty or kyphoplasty. Eur Spine J. 2006;15:1255-64. 